\title{
A case report of incisional hernia through a 5-mm lateral port site following laparoscopic right ovarian cystectomy
}

\author{
A. Sayasneh $\cdot$ H. Nosib $\cdot$ H. Abdel-Rahman
}

Received: 29 October 2008 / Accepted: 29 December 2008/Published online: 22 January 2009

(C) Springer-Verlag 2009

\begin{abstract}
We report a case of postoperative bowel obstruction with early onset hernia presenting through a 5-mm port. A 43-year-old woman having undergone laparoscopic ovarian cystectomy 2 days before presented with features of intestinal obstruction in the form of persistent abdominal pain, vomiting, and inability to pass stool or flatus. Computed tomography scan of her abdomen showed collapsed large bowel and a loop of small bowel protruding through the lateral $5-\mathrm{mm}$ sheath defect. A secondary laparoscopic procedure allowed both confirming diagnosis and surgical repair of the hernia. Our case describes this rare complication through a $5-\mathrm{mm}$ lateral port, with complete bowel obstruction, in the absence of any of the recognized risk factors following a straightforward short surgical procedure, performed as an open laparoscopy with the sample retrieved through the $10-\mathrm{mm}$ trocar. Body mass index was normal, with no significant health problems preoperatively.
\end{abstract}

Keywords Port site hernia - Laparoscopic ovarian cystectomy $\cdot$ Small bowel obstruction

Abbreviations
$\begin{array}{ll}\text { USS } & \text { ultrasound scan } \\ \text { CA125 } & \text { cancer antigen 125 } \\ \mathrm{CO}_{2} & \text { carbon dioxide } \\ \mathrm{A \& E} & \text { Accident and Emergency Department } \\ \text { FBC } & \text { full blood count } \\ \text { Us and Es } & \text { urea and electrolytes }\end{array}$

A. Sayasneh $(\bowtie) \cdot H$. Nosib $\cdot$ H. Abdel-Rahman Department of Obstetrics and Gynaecology, Hinchingbrooke Hospital,

Huntingdon, UK

e-mail: sayasneh@gmail.com

$\begin{array}{ll}\text { LFTs } & \text { lever function tests } \\ \text { CRP } & \text { C-reactive protein } \\ \text { CXR } & \text { chest X-ray } \\ \text { AXR } & \text { abdominal X-ray } \\ \mathrm{CT} & \text { computed tomography } \\ { }^{\circ} \mathrm{C} & \text { degree Celsius } \\ \mathrm{mmHg} & \text { millimeters of mercury } \\ \mathrm{mm} & \text { millimeters } \\ \mathrm{cm} & \text { centimeters } \\ \mathrm{BMI} & \text { body mass index }\end{array}$

\section{Introduction}

Trocar site hernia is a rare cause of major morbidity after laparoscopic surgery. It is a common practice to close $10 \mathrm{~mm}$ rectal sheath defect to prevent this serious complication. However, there is not enough evidence to suggest closing 5-mm laparoscopic incisions. In our case, we report a 5-mm lateral port hernia, and we discussed the incidence, etiology, and prevention of this very rare laparoscopic surgery complication.

\section{Case report}

A 43-year-old nulliparous woman was admitted for an elective laparoscopic right ovarian cystectomy. Her body mass index was 26 , she had no known medical problems, and had never undergone abdominal surgery. She had a simple-looking ovarian cyst on USS measuring $5 \mathrm{~cm}$ diameter, and CA125 was 11.

Three ports were used for carrying out the procedure, of which one was $10 \mathrm{~mm}$ and two were $5 \mathrm{~mm}$. The $10-\mathrm{mm}$ 


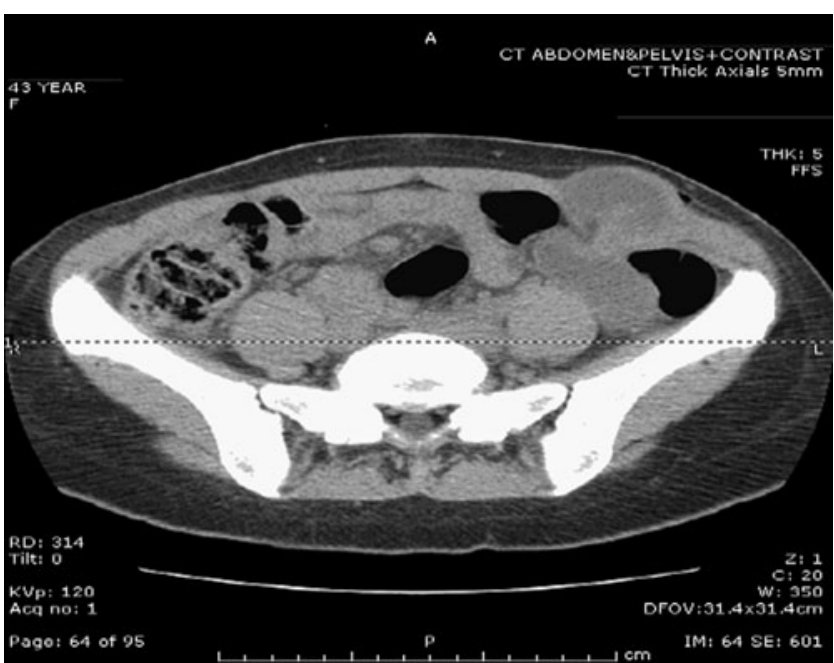

Fig. 1 Small bowel hernia through left 5-mm port site on CT scan

port was an umbilical camera port, and the two $5 \mathrm{~mm}$ were working ports. A Hasson entry was performed, and a 10-mm umbilical port was introduced. Carbon dioxide was insufflated to a pressure of $20 \mathrm{mmHg}$ and then lowered to $15 \mathrm{mmHg}$ when all trocars were in the abdominal cavity. Two 5-mm lateral pyramidal-tipped trocars were inserted on each lower abdominal quadrant outside the rectus sheath under direct vision. Insertion of all ports was obtained at first attempt, with one trocar pass for each port, and no stretching of port sites took place during the procedure. The cyst was dissected using a laparoscopic grasper and retrieved in an endobag via the $10-\mathrm{mm}$ umbilical port under direct vision from the right lateral port. The procedure was uncomplicated; hemostasis was achieved with bipolar diathermy. The lateral ports were removed under direct vision, and the $\mathrm{CO}_{2}$ was expelled through the ports. The umbilical port was removed after complete evacuation of $\mathrm{CO}_{2}$. The umbilical rectus sheath was closed with vicryl 1 . The skin was closed with interrupted monocryl in the umbilical and lateral ports.

On the third postoperation day, she presented to $\mathrm{A} \& \mathrm{E}$ with features of intestinal obstruction in the form of persistent abdominal pain, vomiting, and inability to pass stool or flatus. Her general condition was average, and she was conscious. Her temperature was $37.4^{\circ} \mathrm{C}$, blood pressure was $123 / 67 \mathrm{mmHg}$, and pulse was $70 / \mathrm{min}$. Her abdomen was non-distended and soft. A $4-\times 5-\mathrm{cm}$ hard lump was palpated under the left 5-mm port site and was tender and non-reducible. There were no erythematic changes, local temperature fluctuation, or any other sign of infection at any of the trocars' sites. Bowel sounds were negative. Blood tests including FBC, Us and Es, LFTs, amylase, and CRP were normal. CXR was normal and showed no signs of perforation. Erect AXR showed multiple fluid levels in the small bowel with no gas in the rectum. Surgical opinion was sought, and an urgent CT scan of her abdomen on the same day showed collapsed large bowel and an ovoid area of fluid within the abdominal wall musculature immediately lateral to the left rectus measuring $2.5 \times 4.5 \mathrm{~cm}$ with an internal structure resembling bowel. This confirmed the diagnosis of incisional hernia (Fig. 1).

\section{Management}

She was immediately taken to the theater for laparoscopy/ laparotomy for exploration and reduction of the hernia with possible bowel resection depending on the viability of the herniated bowel. She was maintained on intravenous fluids only, and a nasogastric tube was inserted, which drained 11 of bilious fluid. A urinary catheter was also inserted.

The operation was performed jointly by the general surgical and the gynecology teams. The previous umbilical incision was reopened bluntly, and a scope was inserted through with $\mathrm{CO}_{2}$ insufflation. A small bowel loop was found to have herniated through the left lateral port (see Figs. 2, 3, and 4).The right lateral port was reentered under laparoscopic guidance, and the left lateral port site was enlarged to $3 \mathrm{~cm}$ externally. The herniated bowel was reduced gently through the fascial defect using a finger, applying downward pressure from the skin incision on the left side. The herniated loop of bowel was dusky after reduction but looked well perfused and viable after irrigation with warm saline and observation for $20 \mathrm{~min}$. The rest of the bowels and pelvis looked normal. Bowel resection was not deemed necessary. All the extra $\mathrm{CO}_{2}$ was expelled, and all the port sites were closed with prolene to the sheath and monocryl to the skin.

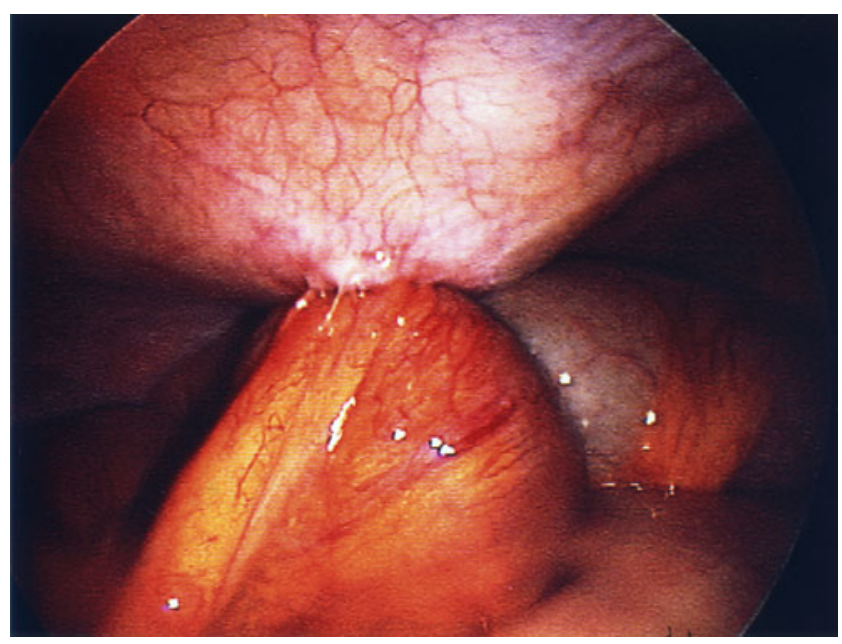

Fig. 2 Small bowel hernia through left 5-mm port site as seen in laparoscopy. Note that part of the mesentery is included in the hernia as well 


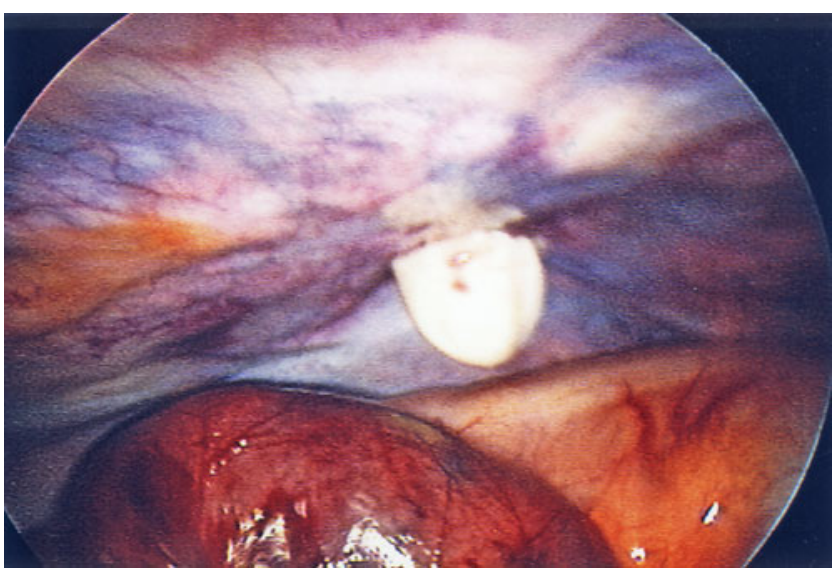

Fig. 3 Small bowels reduced through the old 5-mm port site assisted by the surgeon's index finger, which is seen in the image. Note the dark color, early incarceration changes of the reduced bowel

The patient made a good postoperative recovery. Her initial symptoms settled, and she was discharged home 2 days after her operation with normal bowel function.

\section{Discussion}

The incidence of trocar site hernia is estimated to be between $0.65 \%$ and $2.80 \%$ [1]. In a survey of the American Association of Gynecologic Laparoscopists, post-laparoscopy incisional hernias occur at an approximate incidence of 21 per 100,000 and are associated with significant morbidity [2]. The same survey revealed that $86.3 \%$ of herniae occurred in sites where the trocar diameter was $10 \mathrm{~mm}$ or more, and umbilical herniae were found in $75.70 \%$ as compared to lateral hernias in $23.70 \%$ [2]. In another retrospective study of 320 cases, the prevalence of trocar site herniation after laparoscopic fundoplication was minimal at 3\% [3]. All herniae occurred at the midline supraumbilical port [3].

There are several factors implicating the etiology. They include large trocar size, incomplete closure of fascia at the trocar site, midline trocars, stretching of the port site for organ retrieval, the effect of a partial vacuum while port withdrawal, obesity, poor nutrition, and operation site infection [1, 4]. Reardon et al. [5] suggested in their paper that hernia may also be caused by repetitive motions in different directions during lengthy laparoscopic procedures. These motions may cause significant stretching of the port site, hence allowing a hernia to develop to a considerable size [5]. In the series by Mayol et al. [6], the incidence of port site hernia was higher in closed than in open (Hasson) technique.

There are very few similar reported cases. Tonouchi et al. [1] classified trocar site herniae into three main groups. The first group, where our case falls into, is early-onset hernia, which indicates dehiscence of anterior fascial plane and peritoneum [1]. The late-onset type develops several months after laparoscopy and contains the peritoneum as a hernia sac [1]. The special type indicates dehiscence of the whole abdominal wall [1].

Sirito et al. [7] described a similar complication after laparoscopic ovarian cystectomy; however, the hernia developed a year after the surgery and contained omentum.

Bourdel et al. [8]. reported a Richter's hernia, where only part of the bowel's wall is herniating with no complete obstruction, presenting through a $5-\mathrm{mm}$ sheath incision after laparoscopic hysterectomy.

Our case describes this rare complication through a 5-mm lateral port, with complete bowel obstruction, in the absence of any of the recognized risk factors following a straightforward short surgical procedure, performed as an open laparoscopy with the sample retrieved through the $10-\mathrm{mm}$ trocar. BMI was normal, with no significant health problems preoperatively. Therefore, would closing the $5-\mathrm{mm}$ port site have prevented this serious complication from happening?

There is no satisfying answer for this question. Crist and Gadacaz suggested that fascial defects created by $5.5 \mathrm{~mm}$ do not require closing [9]. On the other hand, Tonouchi et al. [1] suggested that trocar site hernia may be reduced by closing the fascia in all port sites including lateral ones of $5 \mathrm{~mm}$, and they advocated closing the fascial defect and peritoneum for $5-\mathrm{mm}$ trocar defects if active manipulation has occurred during prolonged procedures. The evidence is clearly conflicting, and perhaps, more research needs to be done before a conclusion can be reached. However, after this particular case, we (the team involved in this case) will adapt closing the fascial defect for all ports of any size and location as a safer option with no significant delay in the operating time.

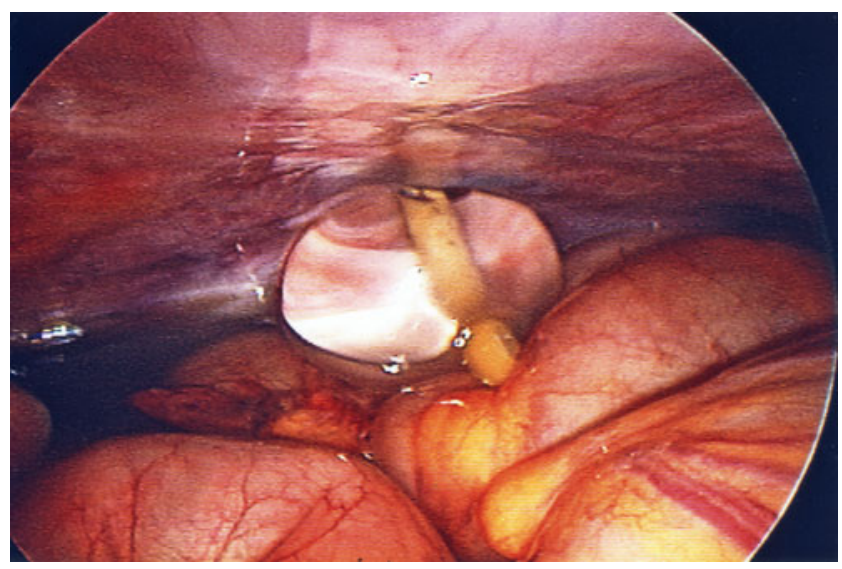

Fig. 4 Twenty minutes after reduction. Note the improved colour of the reduced bowel loop. A Foley catheter balloon was used to prevent gas leakage from abdominal cavity throughout the procedure 
This case has highlighted a very important aspect of laparoscopic surgery: aftercare of patients and prompt recognition and management of postoperative complications. This patient was reviewed promptly by the same team who had performed the operation, and investigations were initiated in a timely way. A thorough review of the patient was performed, and the diagnosis was suspected from the start. This led to appropriate review by the surgical team. Radiology assistance was also readily available, though this all happened out of hours at night. She was treated laparoscopically as expertise was at hand and perhaps avoided an unnecessary laparotomy. She also did not require bowel resection, as the symptoms were seen without delay and theater was arranged for in time. We believe that units performing laparoscopic surgery should have adequate expertise and facilities to deal with potential complications albeit rare at any time, as this significantly alters the prognosis of those involved. Units should have a clear pathway for the referral of patients in the postoperative period for review in case of suspected complications.

Conflict of interest The corresponding author certifies that there is no actual or potential conflict of interest in relation to this article.

\section{References}

1. Tonouchi H, Ohmori Y, Kobayashi M, Kusunoki M (2004) Trocar site hernia. Arch Surg 139:1248-1256

2. Montz FJ, Holschneider CH, Munro MG (1994) Incisional hernia following laparoscopy: a survey of the American association of gynecologic laparoscopists. J Obstet Gynecol 84(5):881-884

3. Bowrey DJ, Blom D, Crookes PF, Bremner CG, Johansson JLM, Lord RV, Hagen JA, DeMeester SR, DeMeester TR, Peters JH (2001) Risk factors and the prevalence of trocar site herniation after laparoscopic fundoplication. Surg Endosc 15(7):663-666

4. Rao P, Ghosh K, Sudhan D (2008) Port site hernia: a rare complication of laparoscopy. Med J Armed Forces India 64:187-188

5. Reardon PR, Preciado A, Scarborough T, Matthews B, Marti JL (1999) Hernia at 5-mm laparoscopic port site presenting as early postoperative small bowel obstruction. J Laparoendosc Adv Surg Tech A 9(6):523-525

6. Mayol J, Garcia-Aguilar J, Ortiz-Oshiro E, De-Diego Carmona JA, Fernandez-Represa JA (1997) Risks of the minimal access approach for laparoscopic surgery: multivariate analysis of morbidity related to umbilical trocar insertion. World J Surg 21:529-533

7. Sirito R, Puppo A, Centurioni MG, Gustavino C (2005) Incisional hernia on the $5-\mathrm{mm}$ trocar port site and subsequent wall endometriosis on the same site: a case report. Am J Obstet Gynecol 193(3 Pt 1):878-880

8. Bourdel N, Roman H, Lebouedec G, Lenglet Y, Accoceberry M, Canis M (2006) Richter's hernia occurring through a 5-mm laparoscopy sheath incision. Gynecol Surg 3:132-133

9. Crist DW, Gadacz TR (1993) Complications of laparoscopic surgery. Surg Clin North Am J 73:265-289 